\title{
Increase of planar homogeneity of multi-silicon structures by gettering treatments
}

\author{
V.G. Litovchenko, A.A. Efremov, A.A. Evtukh, Yu.V. Rassamakin, M.I. Klyui, V.P. Kostylov \\ Institute of Semiconductor Physics, NASU, Kiev, Ukraine, E- mail: ALEF@isp.kiev.ua. \\ Tel./FAX: 044-265-62-90
}

\begin{abstract}
Two types of gettering treatments are considered and compared from the viewpoint of their usefulness to decrease $L_{D}$ scatter over the wafer in multi-silicon photovoltaic structures. It was found that in both cases high degree of homogeneity in $L_{D}$ distribution over the sample surface and cleaning of the samples from recombination active impurities are achieved. Possible mechanisms of the homogenization are briefly discussed.
\end{abstract}

Keywords: gettering, planar inhomogeneity, diffusion length, surface photovoltage.

Paper received 27.03.01; revised manuscript received 29.03.01; accepted for publication 15.05.01.

\section{Introduction.}

The present work is devoted to study of influence of gettering and passivation procedures on planar homogeneity (uniformity) planar distribution of recombination parameter $L_{D}$.

Typically the multi-crystalline silicon ( $\mathrm{m}-\mathrm{Si}$ ) is rather non-homogeneous material as to structural, electrical and photo-electrical properties. This feature is very undesirable for reproducibility, characterization, and for calculations of the basic parameters of photovoltaic solarcell devices.

Therefore, it is very important to find out such types of technological procedures, which will be able to decrease sufficiently the scatter of recombination parameters along the surface of the m-Si wafers used for solar cell fabrication. Besides, suitable approaches for description and characterization of recombination homogeneity have to be developed.

\section{Samples}

The m-Si material, which we deal with, was from two different manufacturers. The two batches of samples were distinguished in bulk oxygen contain:

(i) $\sim 5 \cdot 10^{17} \mathrm{~cm}^{-3}$ for series I;

(ii) $\sim 5 \cdot 10^{16} \mathrm{~cm}^{-3}$ for series II;

Below we shall pay more attention to behavior of samples from the second series.
We have checked usefulness of two different types of gettering and passivation treatments in decrease of the scattering of recombination parameters.

We used ( $i$ ) Al backside getter deposited on m-Si surface and (ii) combined getter when Al layer was deposited on preliminary developed (roughened) Si surface. Gettering treatments were done at $650-1000^{\circ} \mathrm{C}$ for $1 \mathrm{~h}$.

\section{Method for inhomogeneity characterization}

We have measured: ( $i$ ) distribution function for crystallite size along the both surfaces of a wafer determined by optical method and (ii) diffusion length $L_{D}$ distribution function for photo-excited minor carriers $L_{D}=(D \tau)^{1 / 2}$.

$L_{D}$ were measured by means of capacitance photovoltage spectra (Fig. 1) [1]:

$V_{p h} / V_{p h}^{\max }=L_{D} k /\left(1+L_{D} k\right)$

Here $k=k(l)$ is an absorption coefficient as a function of exciting light wavelength. According to ASTM standards approximation [1]:

$$
k(\lambda)=(84.732 / \lambda-76.417)^{2}
$$

where $k$ is in $\mathrm{cm}^{-1}$ and $\lambda$ is in $\mathrm{mm}$.

Therefore, on decaying part of $V_{p h}(\lambda)$ spectrum (approximately from 600 to $1100 \mathrm{~nm}$ ) it is easy to calculate $L_{D}$ and $\tau$, which characterize photosensitivity of semiconductor material . 


\section{V.G. Litovchenko et al.: Increase of planar homogeneity of multi-silicon ...}

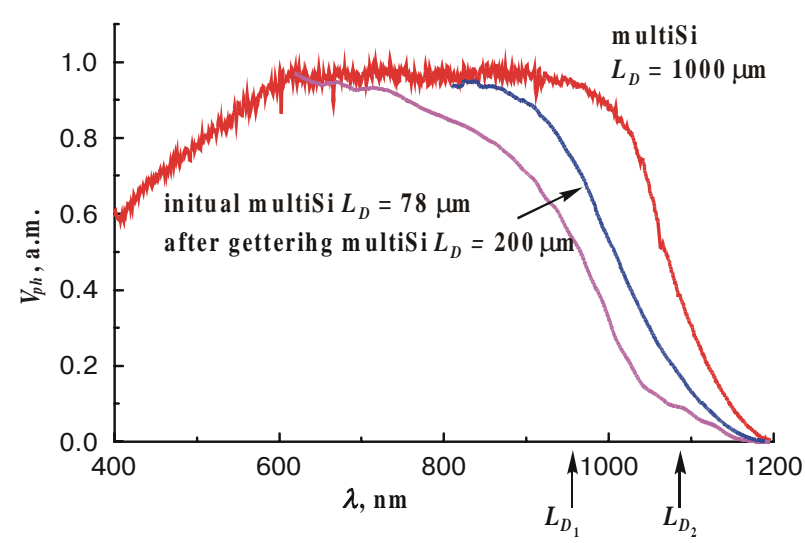

Fig. 1. Spectral dependence for normalized photovoltage for single crystalline silicon $\left(L_{D}=1000 \mu \mathrm{m}\right)$, multi-silicon before $\left(L_{D}=78 \mu \mathrm{m}\right)$, and after $\left(L_{D}=200 \mu \mathrm{m}\right)$ gettering.

Typical view of photovoltage spectra is shown in Fig. 1. The most interesting feature of the $\mathrm{m}$-Si spectrum is the longwave tail caused by heterogeneous distribution of $L_{D}$ over the sample. After gettering treatment this tail decrease remarkably due to homogenization.

\section{Results}

Optical measurements have shown that samples of series I with higher content of oxygen are characterized by about 3 times smaller diameter of crystallites $\left(d_{m} \sim 0.3 \mathrm{~cm}\right)$ than samples from batch II $\left(d_{m} \sim 1.0 \mathrm{~cm}\right)$.

After $L_{D}$ measurements in several points on the wafer surface both average $\left\langle L_{D}>\right.$ and scatter value $\Delta L_{D}$ have been obtained for each wafer.

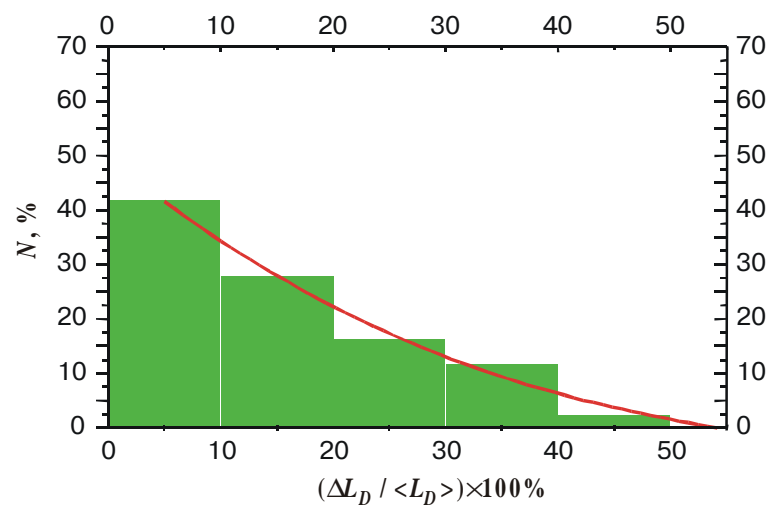

Fig. 2. The general statistical distribution of $\Delta L_{D} /<L_{D}>$ for all samples from Batch II. $\left\langle L_{D}\right\rangle=50.7 \mu \mathrm{m},\left\langle\Delta L_{D}\right\rangle=15.5 \mu \mathrm{m}$, $\Delta L_{D \max }<5 \mu \mathrm{m}$

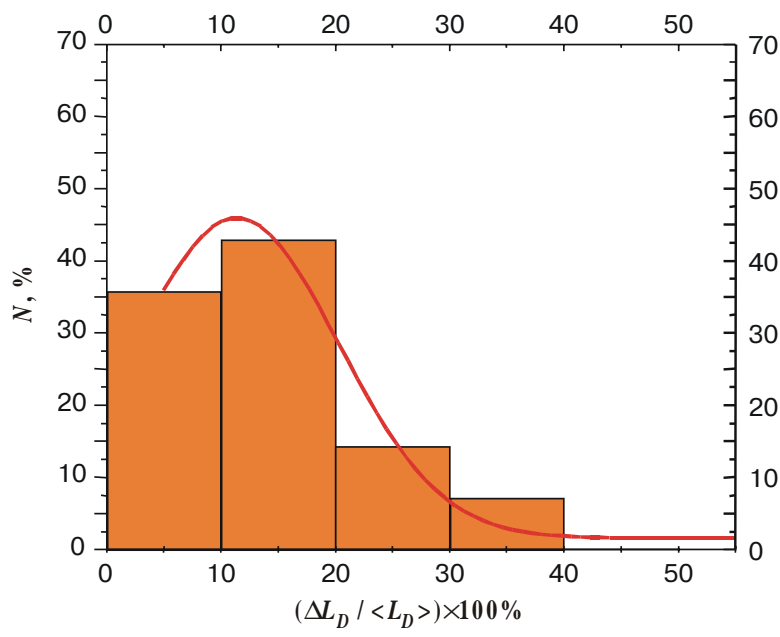

Fig. 3. Special selection \#1 (with combined getter dev-Si $+\mathrm{Al}$ ) prepared for gettering procedure from samples of general selection (Fig. 2). $\left\langle L_{D}>=52.4 \mu \mathrm{m},\left\langle\Delta L_{D}>=14.3 \mu \mathrm{m}, \Delta L_{D \max }=14.3 \mu \mathrm{m}\right.\right.$

$\Delta L_{D}=\left\langle\left|<L_{D}>-L\right|>\right.$

The general statistical distribution of $\Delta L_{D} /<L_{D}>$ for all samples from Batch II is presented in Fig. 2. Than special selections was performed (Figs 3 and 4) from the samples with higher dispersion and subjected to gettering treatments mentioned. One can see that after the both treatments $\Delta L_{D} /<L_{D}>$ ratio decreased sufficiently (Figs 5,6).

\section{Discussion}

In order to understand mechanisms of $L_{D}$ planar inhomogeneity decrease due to gettering treatments, let us outline the main reasons that result in heterogeneity. They are: (i) existence of numerous grain boundaries

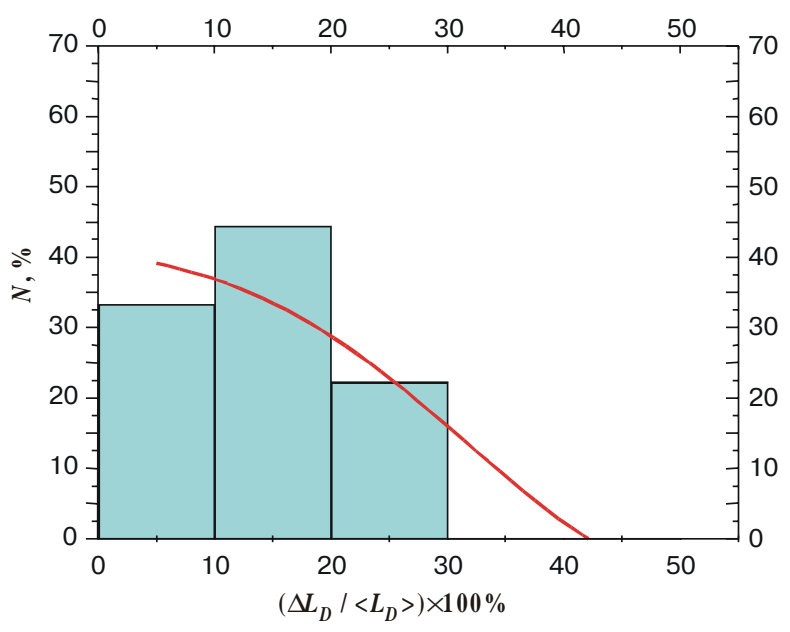

Fig. 4. Special selection \#2 (with $\mathrm{Al}$ ) prepared for gettering procedure from samples of general selection (Fig. 2). $\left\langle L_{D}\right\rangle=$ $=45.1 \mu \mathrm{m},<\Delta L_{D}>=13.9 \mu \mathrm{m} \Delta L_{D \max }=15 \mu \mathrm{m}$. 
V.G. Litovchenko et al.: Increase of planar homogeneity of multi-silicon ...

Table 1. Change of statistical characteristics of $L_{D}$ distribution function

\begin{tabular}{lcccccc}
\hline \hline & \multicolumn{3}{c}{ Initial } & \multicolumn{3}{c}{ After gettering } \\
\hline Series II & $<L_{D}>$ & $\Delta L_{D}$ & $\Delta L_{D} /<L_{D}>$ & $<L_{D}>$ & $\Delta L_{D}$ & $\Delta L_{D} /<L_{D}>$ \\
\hline & $\mu \mathrm{m}$ & $\mu \mathrm{m}$ & $\%$ & $\mu \mathrm{m}$ & $\mu \mathrm{m}$ & $\%$ \\
\hline General & 50.7 & 15.5 & 10 & & & \\
\hline $\mathrm{Al}$ & 45.1 & 13.9 & 15 & 58.2 & 9.4 & 10 \\
\hline Dev-Si+Al & 52.4 & 14.3 & 12 & 91.0 & 10.7 & 12 \\
\hline \hline
\end{tabular}

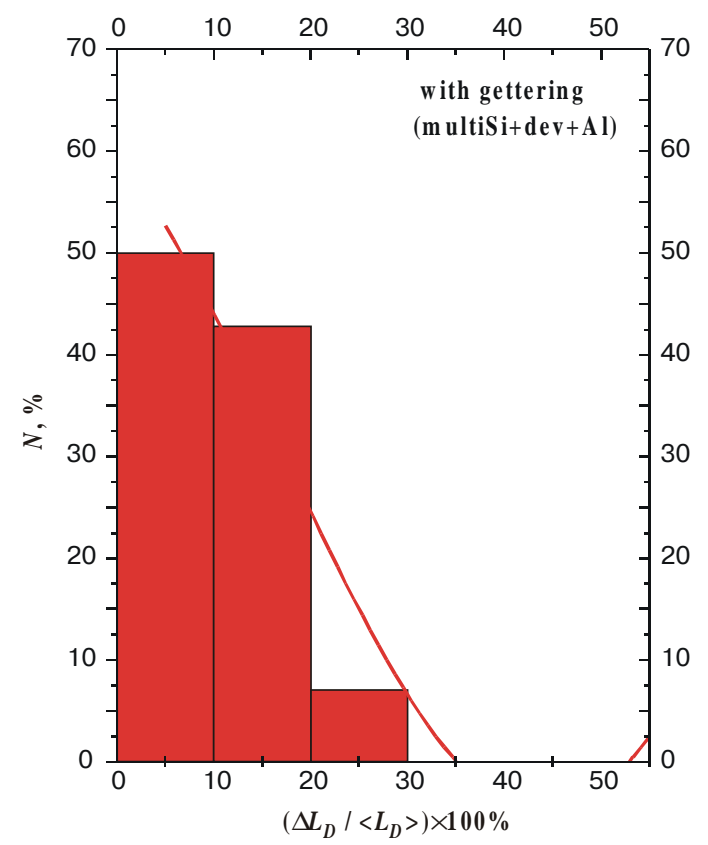

Fig. 5. Special selection $\# 1$ after gettering procedure. $<L_{D}>=$ $=91.0 \mu \mathrm{m},<\Delta L_{D}>=10.7 \mu \mathrm{m} \Delta L_{D \max }<5 \mu \mathrm{m}$.

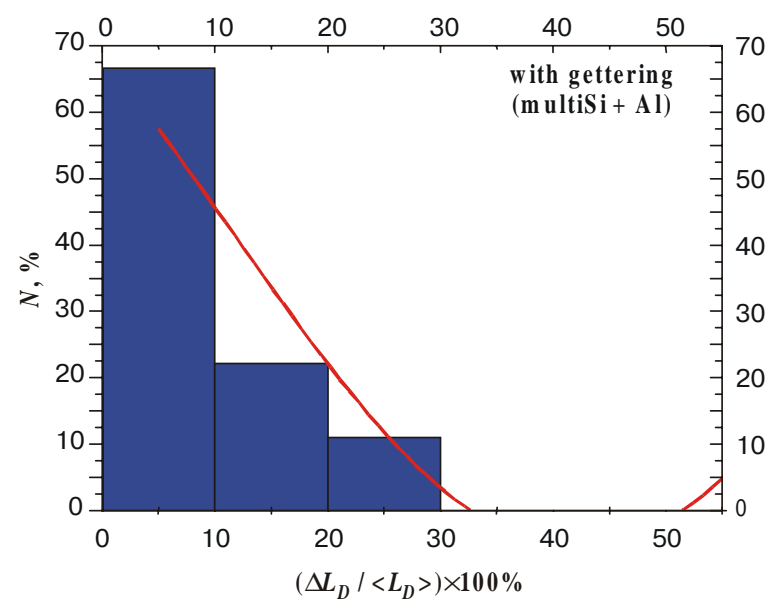

Fig. 6. Special selection $\# 2$ after gettering procedure. $<L_{D}>=$ $=58.2 \mu \mathrm{m},<\Delta L_{D}>=9.4 \mu \mathrm{m} \Delta L_{D \max }<5 \mu \mathrm{m}$. decorated by recombine active impurity atoms with large radius $(\mathrm{Fe}, \mathrm{Cu}, \mathrm{Au})$. These atoms are rather mobile; (ii) existence of $\mathrm{SiO}_{\mathrm{x}}$ precipitates which also have a tendency to be formed in the vicinity of boundary grains. Therefore, in a case of $\mathrm{m}$-Si we have more pronounced fluctuations in spatial distribution of all factors which are responsible for $L_{D}$ suppression.

Thermal treatments result in 3 the most important consequences: ( $i$ ) diffusion induced smoothing in spatial distribution of active impurity (ii) decomposition/growth of initially formed $\mathrm{SiO}_{\mathrm{x}}$ precipitates under silicon interstitials flow (in the case of combined getter) with following re-nucleation of them at nucleation centers in the bulk of grains; (iii) cleaning wafer due to impurity removal and suppression of inhomogeneities in 3D- spatial distribution of impurity atoms.

\section{Conclusion}

It is well known that gettering procedures result in increase of $L_{D}$. We have demonstrated that the mentioned treatments lead to another important gain namely, to sufficient homogenization of $L_{D}$ spatial distribution. If combined getter (dev-Si $+\mathrm{Al}$ ) give more increase in average $L_{D}$ value, the ordinary Al getter proves to be more effective just for suppression of $L_{D}$ spatial fluctuations.

\section{References}

1. Standard Test Methods for Minority Carrier Diffusion Length in Extrinsic Semiconductors by Measurement of Steady State Surface Photovoltage. In: Annual Book of ASTM Standards, Section 10, Electrical Insulation and Electronics, Vol.10.05 Electronics (II), Designation: F 391-96 (1997). 